41 | 2010

Le cheval : monture, nourriture et figure

\title{
Le choix et l'entraînement du cheval de course chez les Kazakhs
}

The selection and training of the racehorse among the Kazakhs

\section{Carole Ferret et Ahmet Toqtabaev}

\section{(2) OpenEdition}

1 Journals

\section{Édition électronique}

URL : https://journals.openedition.org/emscat/1577

DOI : 10.4000/emscat.1577

ISSN : 2101-0013

Éditeur

Centre d'Etudes Mongoles \& Sibériennes / École Pratique des Hautes Études

\section{Référence électronique}

Carole Ferret et Ahmet Toqtabaev, "Le choix et l'entraînement du cheval de course chez les Kazakhs », Études mongoles et sibériennes, centrasiatiques et tibétaines [En ligne], 41 | 2010, mis en ligne le 15 avril 2010, consulté le 13 juillet 2021. URL : http://journals.openedition.org/emscat/1577 ; DOI : https://doi.org/10.4000/emscat.1577

Ce document a été généré automatiquement le 13 juillet 2021.

(c) Tous droits réservés 


\title{
Le choix et l'entraînement du cheval de course chez les Kazakhs
}

The selection and training of the racehorse among the Kazakhs

\author{
Carole Ferret et Ahmet Toqtabaev
}

\section{NOTE DE L'AUTEUR}

Cet article a été écrit par C. Ferret, sur la base de données de terrain et de légendes recueillies par elle et par A. Toqtabaev dans les années 1990, matériaux qu'elle a organisés en y ajoutant des références à des auteurs russes et ses interprétations personnelles, issues de ses recherches doctorales (Ferret 2006).

\section{Les courses de chevaux au Kazakhstan}

1 Au Kazakhstan, les courses de chevaux, dites bäjge ${ }^{1}$, se déroulent traditionnellement sur de longues distances, une vingtaine ou une trentaine de kilomètres, suivant l'âge du cheval. Elles se pratiquent encore de nos jours, désormais sur des pistes circulaires. La saison des courses débute lors de la fête du nouvel an (nauryz²), le 22 mars. Les courses ont lieu au printemps et à l'automne, éventuellement en été dans les régions où le climat n'est pas trop chaud. Dans une même course se mesurent parfois plus d'une centaine de chevaux. Les cavaliers sont des garçons, âgés d'une dizaine à une quinzaine d'années, qui montent le plus souvent à cru et même parfois pieds nus pour être plus légers. Des chevaux des deux sexes - mâles entiers ou castrés, ainsi que des juments participent aux courses et concourent ensemble, bien que, dans l'usage courant, les Kazakhs utilisent seulement des hongres comme chevaux de selle. Outre les épreuves de galop, il existe aussi des courses d'ambleurs (žorǵa žarys), mais les chevaux, les techniques d'entraînement et les cavaliers étant différents, nous ne les étudierons pas ici $^{3}$. 

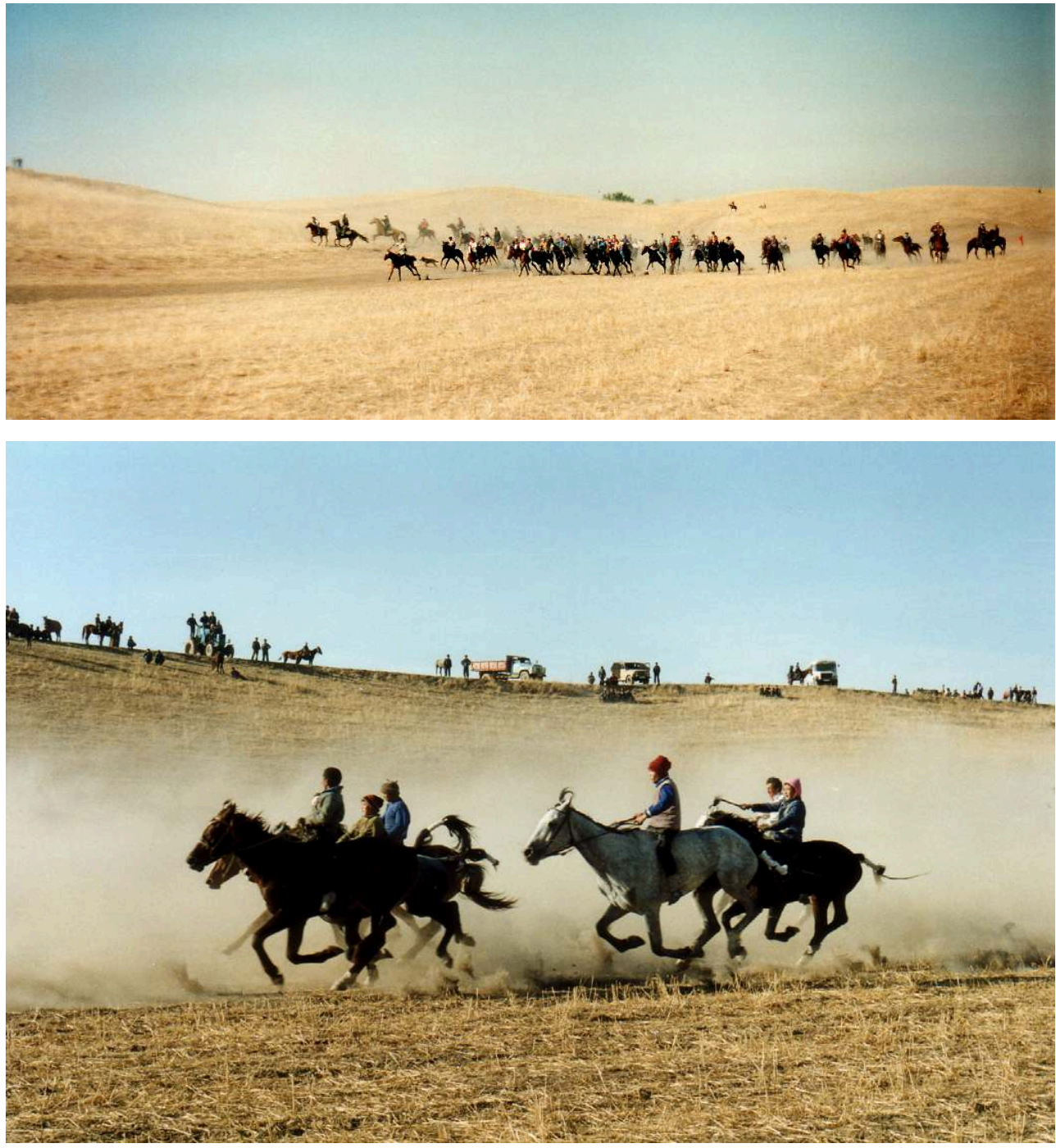

Photographies prises par C. Ferret le 16.10.1994 à Šien, arrondissement de Žambyl, région d'Almaty

La course sur longue distance représente une épreuve au vrai sens du terme pour le cavalier et sa monture. Rares sont les concurrents qui parviennent au but. A l'approche de l'arrivée, les supporters de chaque cheval avaient parfois le droit de pratiquer le köterme $e^{4}$, qui consiste à pousser, tirer, ou même porter le cheval jusqu'au poteau final. Les chevaux de course doivent avoir subi une préparation spécifique dont l'absence ou l'insuffisance peut être fatale. Il arrive que des chevaux mal entraînés succombent lors de la course ou après celle-ci, leur cœur n'ayant pas supporté l'effort consistant à maintenir un galop soutenu sur quelques dizaines de kilomètres.

C'est sur cet entraînement particulier, totalement inconnu en France, que nous nous proposons d'apporter quelques lumières, ce sujet n'ayant, à notre connaissance, fait l'objet d'aucun travail, hormis un article écrit en russe à la fin du xixe siècle (Kaller 1885). Nous avons interrogé, séparément ou conjointement, dans les années 1990, plus d'une vingtaine d'entraîneurs kazakhs âgés de 24 à 90 ans, issus principalement de la grande horde ${ }^{5}$ et habitant dans les régions ${ }^{6} \mathrm{~d}^{\prime}$ Almaty, de Semej - rus. Semipalatinsk -, du Sud kazakh, de Žambyl, de Taldy-Qorǵan, de Pavlodar, d'Aqtöbe - rus. Aktjubinsk -, de Qyzylorda et de l'Est kazakh. 


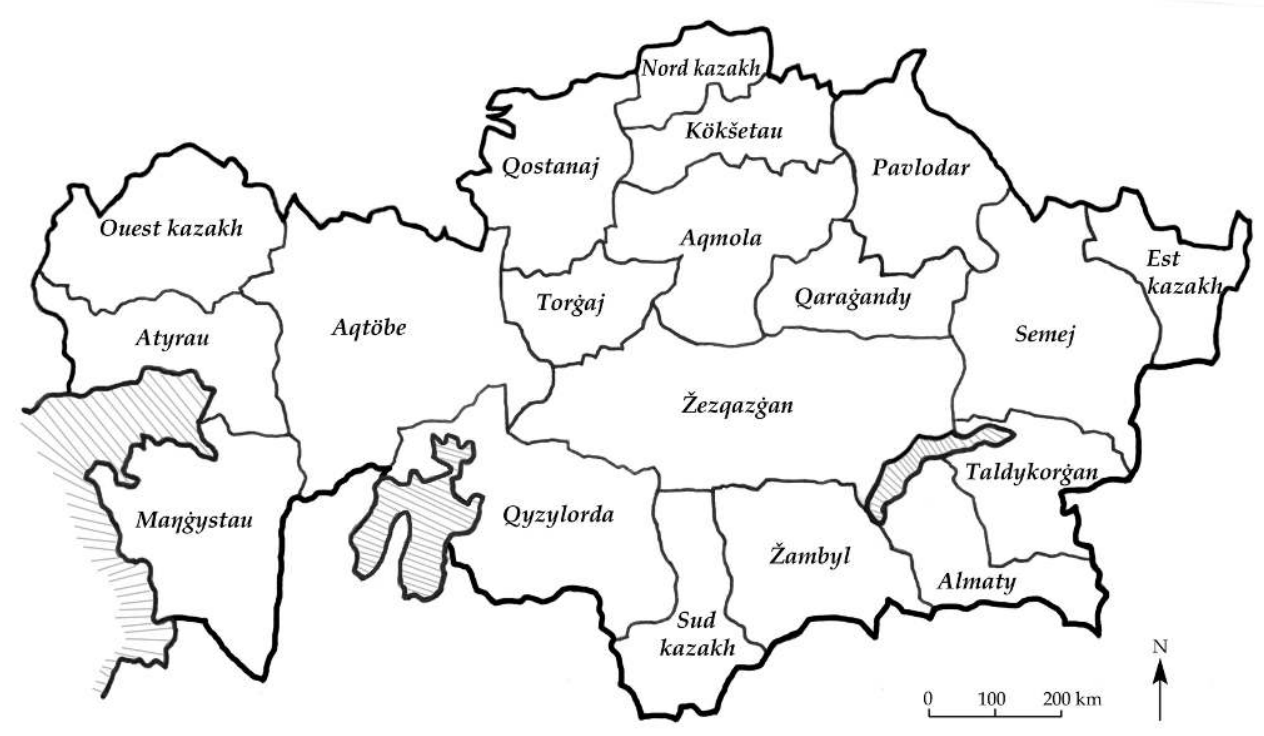

Les bäjge sont des manifestations de prestige, traditionnellement destinées à marquer tel ou tel événement : commémoration d'un mort (as), fête organisée à l'occasion d'une circoncision ou d'un mariage (toj). Au XIX siècle, on organisait des bäjge à l'occasion de fêtes religieuses ou familiales (Dobrosmyslov 1895, p. 122). Depuis les années 1880, elles coexistent avec les courses classiques de courtes distances empruntées à l'Occident, dans un équilibre mouvant qui manifeste les relations entre colonisateurs et colonisés et traduit l'évolution économique, sociale et politique du pays (Ferret 1997, 2008, 2009b).

\section{L'art de l'entraînement et ses spécialistes}

Les bäjge connaissent un renouveau depuis l'indépendance du Kazakhstan (proclamée en décembre 1991), mais, contrairement à ce qu'on entend dire parfois, elles n'ont jamais disparu sous le régime soviétique. Aussi les méthodes d'entraînement traditionnelles se sont maintenues, leurs secrets se transmettant de génération en génération. De nos jours, on rencontre en plusieurs régions du Kazakhstan des maîtres en la matière, qui préparent les chevaux aux compétitions. Il existe deux personnages spécialistes des courses de chevaux. D'une part, les entrâneurs proprement dit, ceux qui savent doser nourriture, abreuvement, travail et repos en fonction des caractéristiques de chaque cheval et qui sont aujourd'hui également éleveurs et propriétaires des chevaux qu'ils entraînent. Les entraîneurs sont nommés atbegì dans le Centre et l'Est, at sejne ou sejne dans l'Ouest, bapker au Sud. D'autre part, les connaisseurs, ou synšy, n'entraînent pas personnellement, mais possèdent le don de pouvoir juger la qualité des chevaux et prédisent avec une exactitude troublante l'ordre d'arrivée aux courses. Ils évaluent les qualités et les défauts d'un cheval au premier coup d'oeil, mais aussi devinent son origine, s'il a été élevé dans la montagne ou dans la plaine, s'il doit courir sur de courtes ou longues distances, etc. Quelques rares individus peuvent même prédire quel genre de poulain se trouve dans le ventre d'une jument et quelle sera sa robe. 
6 Il arrive naturellement que le synšy et l'atbegì ne fassent qu'une et même personne. Dans les années 1860 et 1870, le monde des courses de la horde de Bökej ${ }^{7}$ fut dominé par le personnage d'Aukatym qui, non seulement prédisait avec exactitude les victoires et le destin des poulains, mais achetait aussi lui-même à bon marché des poulains d'aspect minable pour en faire d'excellents coursiers. Un autre synšy, nommé Abilgazy, sauva du couteau du boucher un poulain isabelle aux yeux vairons âgé d'un an et demi, qui fut par la suite invaincu en course (Babadžanov 1871, $n^{\circ} 7$, p. 70). Mais on distingue toujours nettement les fonctions de synšy et d'atbegì : en matière d'entraînement, il y a ceux qui font et ceux qui savent.

\section{La sélection du cheval de course}

7 Les Kazakhs divisent les coursiers en trois types décrits par A. I. Dobrosmyslov (1895, p. 69). Le qargyšyl, cheval «bondissant $»^{8}$, est bon coureur sur de courtes distances (jusqu'à $8 \mathrm{~km}$ ), qualité à laquelle les Kazakhs n'accordent aucune valeur. Le qašaġanšyl, cheval « fuyant $»^{9}$, rapide, mobile et intelligent, est utilisé par les gardiens de troupeaux pour capturer des chevaux avec un qưryq (perche munie d'une boucle non coulissante utilisée pour la capture des chevaux déjà dressés), un tůzaq (lasso) ou un bůgalyq (perche munie d'un noeud coulant, utilisée pour la capture des chevaux non encore dressés) ${ }^{10}$. Enfin le žüjrik bäjge aty, ou coureur de bäjge, le plus prisé de tous et le plus cher, a un galop rasant et allongé, qui allie vitesse et endurance, et il fait la fierté du clan qui le possède. On peut aussi dire de certaines personnes qu'elles sont des žüjrik, cela signifie qu'elles ont une position sociale éminente, qu'elles sont un sujet d'orgueil pour leur famille et leur lignage. En ce qui concerne les chevaux, il s'agit bien de types et non de races. Des représentants de tel ou tel type peuvent apparaître dans n'importe quel groupe de chevaux.

8 La notion de race, typiquement occidentale et relativement récente, a été introduite par les Russes au Kazakhstan. Au xixe siècle, les Russes se désolaient de voir que les Kazakhs ne se souciaient guère d'améliorer leurs chevaux. "Avant que les Russes n'organisent des courses d'État, les Kirghiz ${ }^{11}$ castraient sans pitié les étalons qui s'étaient révélés bons coureurs et ne faisaient pas reproduire leurs juments afin de les utiliser le plus longtemps possible pour les courses » (Babadžanov 1871, n 7, p. 84). Et, quand ils mettaient néanmoins des chevaux de course à la reproduction, ils ne se préoccupaient pas d'accoupler des juments et des étalons de la même qualité sportive. La naissance d'un coursier dans un troupeau était considérée comme un don du ciel, impossible à provoquer par un travail de sélection. On reconnaissait bien un rôle à l'hérédité (tout en accordant plus d'importance à l'étalon qu'à la jument), mais on savait aussi que, comme dit le proverbe, Bìr anadan ala da, qưla da tuady, « la même mère donne naissance à un pie [un mauvais] comme à un louvet [un bon] ». On ne jugeait pas l'étalon reproducteur à son extérieur, lui demandant surtout d'être fort et peu exigeant sur la nourriture (Džantjurin 1883, p. 23). Quelques juments sont engagées dans des courses avant de pouliner, mais les épreuves ne constituent pas, comme en Occident, une manière systématique de sélectionner les bons reproducteurs. L'homme ne peut pas produire le cheval de course, il ne peut que le repérer, et encore seulement s'il a un don de synšy.

9 L'essentiel du discours élaboré sur la sélection du coursier est donc constitué d'une énumération de critères plus ou moins variables, permettant de distinguer l'individu 
qui, après avoir fait l'objet d'un entraînement particulier, pourra aller plus vite et plus loin que les autres. Afin de le soumettre d'emblée à une préparation appropriée, il est préférable de déceler le futur crack dès son plus jeune âge - sa première année si possible. Les signes auxquels on le reconnait sont les suivants : dès la naissance, les naseaux sont grand ouverts ; à l'attache, le poulain se singularise par une mobilité et une vivacité excessives, en même temps que par un comportement doux avec l'homme ; il se laisse bien conduire, il n'est ni ombrageux ni rétif. Mais c'est surtout à son extérieur que, selon les Kazakhs, on voit le bon coursier.

\section{Les beautés du cheval de course}

Un jour, le jeune Tolybaj, longeant le Syr-Daria au petit trot, vit un crâne de cheval sur le bord de la route. Tirant sur les rênes pour arrêter sa monture, il saisit le crâne et se mit à réfléchir : c'était selon toute apparence la tête d'un excellent coursier. Le chanfrein était busqué comme celui de l'antilope, les orbites profondes, la paupière droite, les parotides noires, l'auge large entre les deux joues. Les dents brillaient comme des pierres précieuses. Il déduisit de la denture que le cheval était mort à l'âge de neuf ans... Il frappa le crâne du manche de son fouet, il résonna clairement. Et quel os de qualité! En regardant son chanfrein, il devina que ce cheval avait le garrot haut, les jambes longues, des articulations de lièvre permettant de formidables bonds, qu'il pouvait galoper sans fatigue sur de longues distances... Après avoir fixé le crâne au troussequin de sa selle, Tolybaj se mit à rechercher qui en avait été le propriétaire. Voyant un crâne accroché à sa selle, les gens pensaient qu'il était fou. Il passa presque une année sur les rives du Syr-Daria et finit par trouver ce qu'il cherchait sur le Qaratau. Il s'avéra que ce crâne avait appartenu au fameux cheval Qůndyzqara ${ }^{12}$, dont le maître était un certain Maldybaj. Qůndyzqara avait péri quelques années auparavant en tombant dans un ravin, après être arrivé premier lors d'un raid de vingt-quatre heures, auquel cent chevaux avaient participé. Tolybaj suspendit le crâne à un arbre ${ }^{13}$ et demanda à Maldybaj si Qůndyzqara avait laissé une descendance. Il répondit qu'il n'avait pas de successeur digne de lui et qu'il ne lui connaissait qu'un produit, un poulain galeux de trois ans. Tolybaj se chargea de soigner ce poulain, et il l'engagea dans sa première course alors qu'il avait quatre ans. Ce Čymkara ${ }^{14}$, descendant de Qůndyzqara, remporta constamment toutes les épreuves du Qaratau et du Syr-Daria pendant une quinzaine d'années.

Cette légende du synšy Tolybaj montre l'importance accordée à la tête du cheval, non parce que ses caractéristiques influencent directement les performances sportives, mais parce qu'elle est le reflet du corps tout entier. On peut juger de l'extérieur du cheval d'après les proportions de son crâne. Selon les entrâneurs kazakhs, la forme de la tête doit répondre aux normes suivantes: elle est petite, sèche, les branches du maxillaire inférieur sont suffisamment écartées pour que l'auge ait un pouce de largeur dans sa partie la plus étroite, les ganaches ne sont pas trop épaisses, les naseaux sont larges, les yeux saillants, grands comme ceux du chamelon et brillants comme des charbons ardents. La nuque est musclée. Le front est large et légèrement bombé. Les salières sont profondes d'une phalange, car la sueur du cheval échauffé s'y accumulera sans couler dans les yeux. Les lèvres sont grandes et, en particulier, il est bon que la lèvre inférieure soit pendante, comme dit le proverbe $\mathrm{Er}$ - můryndy, at - erìndì bolsyn, "le cheval se juge à ses lèvres comme l'homme courageux à son nez ", ou plutôt "le cheval est lippu comme l'homme courageux a un grand nez ». Les oreilles sont droites, fermes, mobiles, fines et pointues comme des roseaux. On doit pouvoir placer un pouce dans l'espace qui sépare les incisives des molaires. Enfin le coursier se distingue des 
chevaux ordinaires par la présence d'orifices dans le conduit lacrymal. Selon nos informateurs, ces trous sont au nombre de trois et jusqu'à sept chez les chevaux d'exception.

L'extérieur idéal est le suivant: l'encolure est de longueur moyenne; la crinière, clairsemée; le garrot, haut; le dos, légèrement concave, mais pas ensellé (qaqpanbel " concave comme un piège »), court car un cheval au dos long se fatigue vite. Le rein est court, mais souple et musclé. L'éminence dite en français «bosse du saut ${ }^{15}$ est aussi appréciée par les Kazakhs. La croupe est bien inclinée, presque avalée ${ }^{16}$. La queue est attachée haut, les crins sont rares et fins, les muscles caudaux, extrêmement élastiques. En tirant la queue sur le côté, on doit atteindre la pointe de la hanche avec la dernière vertèbre. Les côtes sont larges et longues; dans le cas contraire, elles sont dites « de serpent ", ce qui est un critère favorable pour les courses de courtes distances, mais pas pour les bäjge $e^{17}$. La poitrine est large et profonde. Pour apprécier sa largeur, on mesure la distance séparant les deux genoux lorsque les quartiers des deux sabots antérieurs se touchent (de préférence supérieure à quatre elì "travers de doigt ", soit 7-8 cm). La distance qui sépare le sabot du coude doit être égale à celle qui sépare le coude du dessus du garrot. L'épaule est musclée, l'angle entre le scapulum et l'humérus doit être obtus. Les ars sont larges et musclés. Les antérieurs ont les muscles et les tendons saillants et bien détachés des os (on doit pouvoir placer trois doigts dans le creux du canon) ; les genoux sont durs et secs ; les châtaignes, dures, avec la pointe dirigée vers le bas; les paturons, droits et courts (le creux du paturon n'a pas plus de deux doigts de largeur) ; les fanons, longs ; les sabots, larges et de forme arrondie ; les jarrets, larges, secs et non droits, la partie antérieure de l'articulation formant un angle pas trop ouvert. Les deux postérieurs sont bien écartés, suffisamment pour qu'un homme puisse passer entre eux et, vus de dos, ils doivent cacher les antérieurs.

Cette énumération de beautés n'est pas sans contradictions. Ainsi, certains veulent le cheval compact; d'autres, plus long que haut et fait en descendant ${ }^{18}$. Contre l'avis de la majorité, quelques-uns déclarent que la tête doit être longue. Les uns veulent l'encolure longue et fine; les autres, courte. Certains veulent les coursiers grands; d'autres, petits ; les derniers disent que la taille n'importe pas. Les critères favorables à la course ne rendent pas nécessairement le cheval « beau » au sens esthétique du terme. Il arrive même qu'un champion ne corresponde pas aux critères, qu'il soit laid, trop maigre, avec de mauvais aplombs. On dit alors qu'il « cache son destin », qu'il dissimule comme à dessein sa qualité pour ne pas attirer le mauvais œil. Beaucoup déclarent qu'il n'existe pas de critères établis, afin de mieux affirmer la finesse et la rareté du savoir des synšy.

Le bon coursier se reconnaît aussi à son comportement. Les rares chevaux qui, la nuit, à l'attache, regardent les étoiles (žůldyzg்a qarap tưrady), sont censés être des animaux d'élite. Avec ou sans cavalier, le cheval doit tenir la tête haute au galop. Attaché au piquet ou à la iourte, il prend une forme arquée ; et lorsque quelqu'un survient, il réagit en courbant tout son corps. Il ne doit pas se tenir perpendiculairement à la iourte mais parallèlement à elle; sa tête s'élève au-dessus de la jonction du kerege (treillis qui forme le mur) et des uyq (perche du toit). Quand il se roule par terre, il se retourne six ou sept fois, signe qu'il maittrise bien son corps. Quand il urine, le jet doit atteindre ses antérieurs. Le bon coursier pait face au vent et s'abreuve dans une rivière en se tenant contre le sens du courant. Il se conduit facilement en main, sans opposer de résistance. Il est calme, n'est pas vicieux et ne mord pas. Mais on veut néanmoins qu'il soit très 
mobile, qu'il ne tienne pas en place, « comme un lièvre », car son sang circule mieux et est plus « pur».

\section{Un débourrage précoce}

14 Ayant reconnu les dispositions du futur coursier, l'atbegì élève le poulain en conséquence. Il le dresse alors qu'il n'a qu'un ou deux ans, en le faisant monter sans selle par des enfants, pour ensuite le faire participer à des courses de chevaux d'un an (taj bäjge) ou de deux ans (qưnan bäjge). Les poulains courent sur des distances un peu plus courtes que les chevaux adultes: les taj bäjge font une dizaine de kilomètres, les qůnan bäjge, une vingtaine, tandis que les alaman bäjge font 30-40 km. Les chevaux peuvent participer à des taj bäjge dès leur deuxième automne, alors qu'ils ont un an et demi. Des juges vérifient la denture des chevaux au départ pour s'assurer de leur âge. Les chevaux plus âgés n'ont pas le droit de participer à des taj bäjge, mais les jeunes chevaux peuvent s'aligner avec des adultes. Au xIX siècle, un certain Ajdabol, du clan Šerkeš de la horde de Bökej, entraîna un poulain nommé Zymyraq « Fougueux » qui, à un an et demi, surpassa tous ses adversaires plus âgés sur une course d'environ trentecinq kilomètres et remporta un prix de cent moutons (Babadžanov 1871, $n^{\circ} 7, \mathrm{p} .70$ ). Ces chevaux exceptionnels ont parfois été débourrés dès l'âge de six mois.

Chez les Kazakhs, le débourrage a toujours lieu dès le plus jeune âge, que ce soit pour les chevaux de course ou les chevaux de travail, alors qu'en Europe, seuls les galopeurs sont montés dès leur deuxième année. Au xix siècle, les voyageurs russes et les spécialistes de l'élevage équin jugeaient sévèrement les pratiques kazakhes consistant à traire les juments, à garder les poulains attachés sous un soleil de plomb, à dresser les chevaux très jeunes, à les faire monter par des enfants. Selon eux, ces habitudes nuisaient gravement à la croissance et au développement des jeunes chevaux. Mais les Kazakhs estiment au contraire qu'un cheval de course endurci jeune franchit de plus grandes distances, qu'il est plus résistant et moins capricieux, qu'il a le goût du travail. C. Džantjurin remarque qu'il est rare de voir dans les steppes un cheval qui ne soit pas encore dressé à deux ans, sauf chez les riches propriétaires, mais il estime que ce débourrage précoce, loin de nuire, développe la musculature, rend le cheval fort et infatigable, l'endurcit, le trempe, alors que les chevaux débourrés à trois ou quatre ans deviennent fragiles et mous (1883, p. 24). Aujourd'hui encore, on juge qu'un cheval débourré jeune est plus fort, notamment parce que ses articulations sont plus solides, à condition de réduire son allure en terrain accidenté ou trop dur. En outre, un poulain oppose moins de résistance lorsqu'il est monté pour la première fois. Si sa mère a été traite, il est déjà habitué à être manipulé par les hommes puisque, dans les premiers mois, il a été attrapé et attaché quotidiennement pour la traite. Le débourrage se déroule tout simplement, sans susciter de problème particulier pour le cavalier ni de traumatisme pour le cheval. Après avoir été monté pendant l'été par des enfants, le poulain est relâché dans les pâturages à l'automne et on ne le monte plus guère l'année suivante, alors qu'il a trois ans.

$16 \mathrm{Au}$ XIXe siècle, un célèbre synšy nommé Küreybaj, du clan Arg்yn de la horde moyenne, précisait :

Monte ton poulain d'un an comme un poulain d'un an

N'épargne pas ton deux ans, presque jusqu'à le tuer

Laisse libre le trois ans 
Engage le quatre ans aux courses

S'il n'arrive pas premier, tu peux me trancher la tête.

Tous les entraîneurs ne suivent pas ces conseils, mais ce dicton montre que la précocité du débourrage ne se justifie pas uniquement par des raisons économiques, puisque en " laissant libre le trois ans », on perd une année de travail. L'idée sous-jacente est qu'il existe des âges favorables et des âges maudits (trois et six ans), des époques propices au travail, d'autres au repos. Interrompre le travail une année n'est pas dommageable, car le cheval n'oublie pas l'apprentissage qu'il a subi. Certains chevaux ne débutent en courses qu'après l'âge critique des trois ans. Dans ce cas, l'entraînement de la première saison est plus délicat et requiert plus d'attention. Par la suite, il sera plus facile d'année en année, si on ne commet pas d'erreur. Malgré ce débourrage précoce, les chevaux de courses kazakhs se distinguent par leur longévité : certains participent à des bäjge jusqu'à quinze ans passés, s'ils sont bien soignés et convenablement entraînés.

\section{L'axiome de l'attache}

Les deux éléments fondamentaux dans l'entraînement du cheval de course sont la nourriture et l'exercice. L'art de l'entraîneur consiste à bien doser l'un et l'autre pour chaque cheval et à faire évoluer ce dosage au cours des phases de préparation afin d'amener le cheval à sa meilleure forme pour le jour J. En dépit de leurs variations, les méthodes d'entraînement présentent certaines constantes. Quelles que soient les régions et les époques, les entraîneurs s'accordent sur quelques grands principes auxquels on ne peut déroger. Tout d'abord, les deux éléments fondamentaux de l'entraînement, la nourriture et l'exercice, ne doivent pas se côtoyer : le cheval ne doit pas être alimenté ni abreuvé dans les heures qui suivent le travail. Ce principe vaut non seulement pour les chevaux de course kazakhs, mais aussi pour tous les chevaux des steppes eurasiennes, indépendamment de leur utilisation. Or le moyen le plus sûr d'empêcher un cheval de consommer quoi que ce soit est de l'attacher. Quand on parle de cheval à l'attache, il faut donc comprendre qu'il n'est ni nourri ni abreuvé. Il est attaché à un poteau, ou parfois à une corde entre deux poteaux, ce qui lui donne le loisir de marcher un peu, mais non de brouter (Barmincev1958, p. 54). Attacher un cheval n'est pas seulement réduire sa mobilité, mais plus encore lui interdire toute pitance.

Tout cheval doit rester attaché sans nourriture ni abreuvement pendant plusieurs heures après le travail sous peine d'être irrémédiablement gâché. Un cheval qui, en une occasion, n'a pas été convenablement attaché après avoir fourni un effort important et qui, par exemple, a bu beaucoup d'eau froide avant d'être sec, devient qyzyl maj «(à) graisse rouge " tare pratiquement incurable. Même bien nourri, il ne sera jamais en état et maigrira dès qu'il travaillera un peu. Selon Benkevič, un cheval qyzyl maj se reconnaît à ce qu'il mange mal, qu'il sue abondamment et maigrit rapidement, que son poil est hérissé et perd son brillant. On arrive parfois à corriger ce défaut en faisant suer l'animal, puis en le gardant attaché très longtemps (1905, p. 1034). Un cheval peut aussi devenir qyzyl maj s'il a soudain fourni un trop gros travail alors qu'il n'était pas en condition, qu'il était gras et sédentaire. C'est pourquoi les Kirghiz, parlant d'un cheval insuffisamment entraîné ayant péri d'épuisement lors d'une course, disent également : kyzyl maj bolup kalgan « il est tombé kyzyl maj » (Simakov 1984, p. 83). Cette affection est parfois fatale, mais on peut tenter de la soigner en faisant boire du thé vert à la bête 
atteinte. Quelle que soit la cause qui rende le cheval qyzyl maj, l'interprétation fait intervenir les catégories du chaud et du froid : le cheval a été gâté (bůzylġan at « cheval abîmé ») par le contraste brutal du chaud (l'exercice) et du froid (l'eau fraîche après l'effort) ou par une surchauffe, la chaleur de l'effort venant s'ajouter à celle de la nourriture accumulée sous forme de graisse. Dans ce dernier cas, on le dit aussi iši qyzg்an at "cheval dont les entrailles ont surchauffé $»^{19}$. L'objectif de l'entraînement sera donc d'amener le cheval à la condition physique la mieux adaptée à la course tout en respectant cet impératif universel de l'attache.

\section{Viande noire ou viande rouge} totalement méconnue de la littérature spécialisée, est celle qui sépare les chevaux en deux types de constitution : qara ettì « à viande noire » et qyzyl ettì «à viande rouge »ou encore maj ettì «à viande grasse ». Cette distinction n'est pas toujours claire ni dénuée d'ambiguïtés, mais voici comment nos informateurs nous l'ont expliquée.

Tout cheval appartient par nature à l'un des deux types, mais ne transmet pas nécessairement cette caractéristique à sa descendance. On peut reconnaître le type auquel appartient un poulain depuis la naissance jusqu'à deux ans en regardant les principaux points où se loge la graisse chez le cheval : sous la crinière, sur les côtes et le ventre. Le cheval qara ettì est plus beau extérieurement, il a le corps compact et des formes rondes, mais sa viande est dépourvue de graisse et il se fatigue vite. Le cheval qyzyl ettì est maigre d'aspect, mais sa viande est grasse et sa maigreur ne l'empêche pas d'être parfois aussi fort que le premier. Cette division reflète donc deux types de constitution interne et externe, l'une compensant l'autre: celui qui est maigre à l'extérieur est gras à l'intérieur et vice-versa. Comme les constitutions physiologiques de ces chevaux sont différentes, il convient de leur appliquer des méthodes d'entraînement distinctes.

Les chevaux à viande noire sont plus faciles à entraîner pour plusieurs raisons. Ils n'ont pas besoin d'être soumis à un entraînement intensif ; on peut les laisser paître même si on leur donne par ailleurs une nourriture sèche. Leur état d'engraissement est plus visible : chez eux, on définit aisément l'épaisseur du qazy « graisse des côtes ${ }^{20}$; or il est indispensable que l'entraîneur connaisse l'état d'engraissement de son cheval afin de moduler son entraînement en conséquence. Enfin ces chevaux traduisent plus sûrement par leurs résultats les bons soins qu'on leur a prodigués. Pour les entraîner, on se contente d'augmenter progressivement la distance qu'on leur fait parcourir chaque jour. On les arrête dès la première suée "amère " ( $c f$. infra), puis on les fait marcher au pas et on les garde attachés quelques heures. Ensuite, on les laisse paître dans la steppe, et on les nourrit avec deux jointées ${ }^{21} d^{1}$ avoine par jour.

Les chevaux à viande rouge - ou grasse - doivent être soumis à un entraînement plus rigoureux et à de longues séances d'attache, ils ne doivent paitre qu'avec la plus grande modération. Même s'ils semblent maigres et suent moins à l'entraînement après quelques nuits passées à l'attache, cela ne signifie pas que leur graisse interne a suffisamment réduit; on le voit parce qu'après l'exercice, leur poil met longtemps à sécher et devient huileux. C'est pourquoi l'entraînement de ce type de chevaux réclame une attention soutenue et nécessite l'emploi de procédés originaux (attache prolongée, abreuvement particulier, sudation stimulée) que nous verrons par la suite.

Études mongoles et sibériennes, centrasiatiques et tibétaines, 41 | 2010 

Cette distinction entre chevaux à viande rouge et chevaux à viande noire a une fin
uniquement pragmatique et non normative : aucun entraîneur n'estime qu'un des deux
types est supérieur à l'autre, mais simplement qu'il faut les entraîner différemment.

\section{Pâture ou aliments secs?} moment où l'on capture le cheval dans les pâturages pour le ramener à la maison marque le début de l'entraînement. Selon Kaller, le cheval de course mâle, après la saison des courses de printemps, se voit confier un troupeau de juments jusqu'à l'automne. Quant aux juments, on les met aussi au vert, mais en attendant la fin de la saison de monte pour qu'elles ne soient pas pleines si on désire qu'elles courent l'année suivante $(1885$, p. 60$)$. Certains entraîneurs envoient les chevaux au pré dès le lendemain de la course pour quelques jours afin qu'ils se reposent, avant de les préparer à la prochaine compétition.

Selon les partisans du pâturage, les chevaux ont besoin de l'herbe verte gorgée d'eau du printemps et le pacage d'été rend leurs os forts et solides, avec une moelle de qualité. Or, pour les Kazakhs, la force du cheval réside dans la moelle de ses os. On ne gagne pas avec ses muscles, noirs ou rouges, mais avec ses os, ou plutôt leur moelle.

Certains entraîneurs recommandent même de mettre le cheval au pré au cours de l'entraînement pour le maintenir en forme, car l'orge favorisant la sécrétion de sueur, elle n'est plus utile une fois que le cheval est en condition. Quelques-uns font paître leurs chevaux le jour de la course, à l'aube, «le temps de préparer un thé » (un quart d'heure), disant que ce court moment de pacage donne au cheval la force nécessaire pour terminer la course. Un entraîneur de l'arrondissement de Kegen met ses chevaux au pré les trois derniers jours précédant la course : ils ne consomment plus que de l'herbe verte et de l'eau et refusent eux-mêmes le foin. Mais la plupart des entraîneurs qui font paitre leurs chevaux de course combinent nourriture sèche et nourriture humide et ils n'autorisent le pacage que pendant une durée limitée, une heure par jour par exemple.

On veille non seulement à la quantité d'herbe ingurgitée mais aussi à la qualité des pâturages. Une légende raconte qu'un jour, dans le bourg d'Aqbastau du Semireč'e ${ }^{22}$, eut lieu un $a s^{23}$ en souvenir d'un certain Qalabek. Un entraîneur dénommé Qůmġanbaj présenta son coursier gris aux joues jaunes à deux synšy, les frères Baqtybaj et Olžabaj. Ce dernier lui dit alors: "C'est dommage, mais ton cheval a perdu ses forces en pâturant ici. Si tu l'avais nourri d'herbe et d'eau de ton pays, il serait arrivé premier. Mais, que faire? Cette fois-ci, il se contentera de la quatrième place ». Ce cheval arriva effectivement quatrième comme l'avait prédit le synšy.

La nature et la qualité de l'herbe jouent un rôle essentiel dans la réussite de l'entraînement. Certaines localités possèdent des pâturages dédiés aux chevaux de course, souvent riches en sparte penné - stipa pennata (kaz. betege). Tôt le matin de la course, un entraîneur de la région de Qyzylorda emmène son cheval paître dans un

Études mongoles et sibériennes, centrasiatiques et tibétaines, 41 | 2010 
endroit où pousse de la fétuque ovine - festuca ovina. Au début du $\mathrm{xx}^{\mathrm{e}}$ siècle, on a remarqué que les chevaux de Sary-Arka (région de Žezqazġan) qui participaient à des courses au Semireč'e ne pouvaient jamais gagner car les alpages de l'Alatau leur causaient des diarrhées. C'est pour éviter ce genre de problèmes qu'on jugeait préférable que les courses débutent sans tarder, dès l'arrivée des chevaux sur le lieu de la fête.

Cependant, nombre d'entraîneurs proscrivent totalement le pacage pour les chevaux de course car ils estiment que l'humidité est nuisible, elle empêche les chevaux de tenir la distance. Interdire le vert est aussi un moyen de prévenir les diarrhées. Leurs chevaux doivent consommer exclusivement une nourriture sèche, peu volumineuse et riche (foin et céréales). Certains mouillent l'avoine avant de la distribuer, mais d'autres adeptes des aliments secs vont jusqu'à légèrement griller l'avoine qu'ils ont lavée pour la débarrasser de toute humidité. Même si les pratiques diffèrent, tous les entraîneurs s'accordent à dire que le pacage fournit au cheval une nourriture humide dont le cheval de course ne doit pas abuser. Entraîner consiste à traquer l'humidité.

\section{Un abreuvement particulier}

Le cheval de course doit être sec, c'est à dire qu'il faut éliminer sa graisse et son eau. On ne peut pas empêcher un cheval de boire, mais certains entraîneurs limitent les quantités d'eau ingurgitées. Un propriétaire de la région de Qyzylorda habitue progressivement l'organisme de son cheval à se contenter d'une petite quantité de liquide. Il appelle ce procédé bìr išek littéralement "un seul intestin», comme si l'intestin avait raccourci au point de ne plus former de circonvolutions; on entend par là un état où le cheval consomme peu et maigrit).

D'autres remplacent l'eau par du lait, qui nourrit en même temps qu'il abreuve. Il permet de compenser le faible apport calorique d'une nourriture distribuée très parcimonieusement. Kaller précise que, si l'avoine vient à manquer pendant l'entraînement, elle peut être remplacée avantageusement par un seau quotidien de lait cru (1885, p. 64), souvent du lait de jument frais (saumal), non encore transformé en kumys, plus rarement du lait de chamelle.

Les Kazakhs considèrent le thé comme un élément purifiant. Näcìbolda Nůrsipov, entraîneur de l'arrondissement de Marqaköl, dans l'Est kazakh, en donne à ses chevaux pour réduire les troubles intestinaux liés à la course. Il les abreuve alternativement avec du thé et de l'eau et, la veille de la course, toujours avec du thé. Si les chevaux refusent de boire du thé, il les y contraint en les assoiffant. Il ne limite pas la quantité de liquide mais déclare que, de lui-même, un cheval à l'entrainement mange et boit peu.

\section{Une nourriture et un exercice calculés}

Composer les deux éléments fondamentaux de l'entraînement consiste à les proportionner harmonieusement. En Occident, on a tendance à penser que, puisque la nourriture se traduit par un gain de calories et l'exercice par une perte de calories, il faut, pour que la comptabilité soit quotidiennement équilibrée, que ces deux éléments demeurent constamment proportionnels l'un à l'autre. Plus un cheval travaille, plus il 
est nourri et, s'il reste au box, sa ration est diminuée. La même opinion existe au Kazakhstan, mais cet équilibre se fait sur un laps de temps plus long: non plus quotidiennement, mais annuellement. L'année du cheval de course se divise en périodes d'engraissement, où le cheval fait peu d'exercice et est nourri abondamment, et en périodes d'amaigrissement, où le cheval, tout en travaillant davantage, est moins nourri. La préparation à la course consiste précisément en une cure d'amaigrissement.

Les périodes d'engraissement correspondent aux moments pendant lesquels le cheval de course n'est pas entraîné et il ne court pas. Cet engraissement peut avoir lieu dans les pâturages à la belle saison (cf. supra), ou à l'écurie pendant la mauvaise saison. L'hiver, Nůrbol Malybekov, entraîneur dans l'arrondissement de Kegen (région d'Almaty), garde son cheval de course à l'écurie. Il ne le fait pas travailler et le nourrit abondamment : tous les jours à $9 \mathrm{~h}$, il l'abreuve puis il lui donne $8 \mathrm{~kg}$ d'orge ; à $11 \mathrm{~h}, 12$ à $20 \mathrm{~kg}$ de foin ; à $17 \mathrm{~h}$, de nouveau il l'abreuve puis il lui donne $8 \mathrm{~kg}$ d'orge et $12-20 \mathrm{~kg}$ de foin. Le cheval ne sort que pour aller boire et est ainsi gavé jusqu'en février d'une manière que des hommes de cheval occidentaux jugeraient ahurissante ${ }^{24}$. D'autres, après la saison des courses qui dure jusqu'à la première neige, distribuent en hiver du grain à volonté, sans plus respecter aucune norme.

On passe ensuite progressivement de la phase d'engraissement à la phase d'amaigrissement. Selon Kaller, de retour des pâturages à la fin de l'automne, le coursier, plutôt gras, est utilisé avec modération comme cheval de selle et nourri de foin, en attendant l'entraînement proprement dit qui commence début mars (1885, p. 60). Aujourd'hui, les chevaux de course ne sont pas, en principe, employés à un travail quelconque, mais, possédant peu de chevaux, la plupart des propriétaires sont amenés à se déplacer parfois sur leur coursier. Après une phase d'inactivité complète, les chevaux sont montés en février une à deux heures par jour, au pas et au trot. Ils sont gardés la nuit à l'écurie, le jour dans un enclos.

L'entraînement, qui correspond à une phase d'amaigrissement, est de durée variable selon la méthode employée, l'état du cheval (plus il est gras, plus l'entraînement est long) et la distance à parcourir. Le jour de la course est habituellement annoncé un mois et demi à deux mois à l'avance, afin de laisser aux concurrents le temps de préparer les chevaux.

39 L'exercice a lieu régulièrement et suit un horaire défini. Les entraîneurs s'efforcent de faire courir plusieurs chevaux en même temps et leur font mener la course tour de rôle pour les habituer à être en tête. Ils s'ingénient à donner aux coursiers des allures régulières, ce qui ne leur est pas naturel. Chaque cheval est monté par le même garçon pendant l'entraînement et le jour de la course, afin que le cavalier et sa monture s'accoutument l'un à l'autre. Il est toujours monté avec une selle à l'entraînement, même s'il l'est à cru pendant la course.

Kaller décrit un entraînement qui débute le $1^{\mathrm{er}}$ mars et dure deux mois et demi. Dans les premiers temps, le cheval reçoit le matin un peu de foin, de l'eau, puis environ $1 \mathrm{~kg}$ d'avoine, puis du foin à volonté (l'ordre de distribution a son importance). L'aprèsmidi, on lui donne de nouveau $1 \mathrm{~kg}$ d'avoine. Le soir, on lui fait parcourir 3 ou $4 \mathrm{~km}$, puis il passe la nuit attaché. En avril, on poursuit le même régime, mais en effectuant la distance au trot. A partir de la mi-avril, on parcourt 1 à $1,5 \mathrm{~km}$ au petit galop, le reste au trot, et on supprime l'avoine du matin, tout en donnant du foin à volonté avant d'abreuver et en continuant de donner $1 \mathrm{~kg}$ d'avoine le soir. Par la suite, le cheval ne travaille plus que tous les trois ou quatre jours. Il est ainsi entraîné à des allures variées 
jusqu'à ce que son ventre ait diminué de volume, puis on passe à la phase finale de l'entraînement comportant des suées (1885, pp. 60-61).

41 Selon Barmincev, le cheval est nourri d'une petite quantité de foin avec une à deux jointées d'avoine ou d'orge. Pendant les premières semaines, il marche au pas 5 à $10 \mathrm{~km}$ par jour. Puis on le fait travailler au trot et au galop, réservant le grand galop aux deux dernières semaines précédant la course (1958, p. 54). Selon Dobrosmyslov, la ration quotidienne du cheval se compose de foin et d'une quantité d'avoine ou d'orge qui diminue progressivement, passant de $2 \mathrm{~kg}$ à $400 \mathrm{~g}(1895, \mathrm{p}$. 127).

Nůrbol Malybekov commence la véritable préparation aux courses en mars. Pendant deux semaines, ses chevaux parcourent quotidiennement 2 à $3 \mathrm{~km}$ à un galop moyen. À 9 et $17 \mathrm{~h}$, ils sont abreuvés et reçoivent $1 \mathrm{~kg}$ d'orge ; pendant la nuit, ils ont du foin à volonté. A partir du 15 mars, la ration du soir est supprimée et la distance du galop progressivement augmentée, jusqu'à $35 \mathrm{~km}$ à la fin du mois. Lors de chaque séance, le cheval galope doucement pendant 1 ou $2 \mathrm{~km}$, puis plus rapidement «à sa propre demande »; il finit par 1 ou $2 \mathrm{~km}$ de galop calme et est rentré au pas ou au petit trot. Peu à peu, le cheval transpire moins au galop, les traces de sueur diminuent sur sa poitrine, sous ses oreilles, entre ses cuisses. Un cheval en forme perd son ventre et mange moins, il ne consomme pas tout le foin qu'on lui donne. Fin mars, il est prêt. En attendant les courses, on entretient sa condition par 1 à $3 \mathrm{~h}$ quotidiennes de trot. Il ne mange plus qu'une fois par jour. Le matin, il est simplement abreuvé et, après l'entraînement, il est attaché jusqu'au soir où il reçoit $1 \mathrm{~kg}$ d'orge, de l'eau et du foin. En mai, on cesse de lui donner de l'orge et on le fait paitre.

43 Žanybek Alybekov, entraîneur à Lugovoj, dans la région de Žambyl, estime la ration journalière à $6 \mathrm{~kg}$ d'orge et 10 à $12 \mathrm{~kg}$ de foin, distribuée en deux fois. On augmente progressivement la durée de l'entraînement quotidien : 1 à $2 \mathrm{~h}$ de décembre à janvier, 2 à $3 \mathrm{~h}$ en février, 4 à $5 \mathrm{~h}$ en mars, en alternant un jour au pas, un jour au trot et au canter, jusqu'à la première course. En été, les compétitions cessent à cause de la chaleur mais l'entraînement continue, tôt le matin ou tard le soir.

Ǵabit Qůraqbaev, entraîneur dans l'arrondissement de Žambyl (région d'Almaty) donne à ses chevaux du trèfle et $1 \mathrm{~kg}$ d'avoine tous les soirs pendant la période des courses ; en temps normal, $1 \mathrm{~kg}$ d'avoine le matin et $1 \mathrm{~kg}$ le soir. Il les entraîne quotidiennement pendant $1 \mathrm{~h}$ à $1 \mathrm{~h} 1 / 2$, doucement au début, quand ils sont gras, car leur coeur ne supporte pas les allures rapides, et plus vivement par la suite. Puis il les attache sans nourriture pendant 4 à $5 \mathrm{~h}$ ( $3 \mathrm{~h}$ pour les plus jeunes).

Les propriétaires veillent scrupuleusement aux quantités et à la nature des aliments, mais aussi à l'ordre de distribution, qui est généralement le suivant : foin - eau - grain. En effet, les aliments sont affectés de différentes valeurs qu'on ne peut mélanger impunément : le foin est un élément «neutre ", dont le cheval ne se gave pas, tandis que l'eau est un élément «froid» et le grain un élément «chaud » ${ }^{25}$. C'est pourquoi, après le travail, qui "échauffe » le cheval, il faut tout d'abord respecter un temps d'attache puis commencer par donner un aliment neutre.

A propos du rapport entre nourriture et entraînement, les exemples présentés permettent de dégager le principe général suivant : augmenter l'exercice (la durée des séances d'entraînement, la distance parcourue, la vivacité des allures), tout en réduisant l'alimentation. Certains entraîneurs jouent en même temps sur l'ensemble de ces paramètres, d'autres sur quelques-uns seulement. Pour accélérer la cure 
d'amaigrissement, et notamment pour les chevaux à viande rouge, on a recours à des procédés stimulant la sudation.

\section{Les bienfaits de la sudation}

La sudation permet d'extirper la graisse et l'eau du corps de l'animal. Et la qualité de la sueur extraite est censée révéler les progrès de l'entraînement. Pour la tester, les entraîneurs goûtent eux-mêmes la sueur de leurs chevaux après l'effort.

Les Kirghiz, qui préparent leurs chevaux de la même manière que les Kazakhs ${ }^{26}$, distinguent différents stades dans l'évolution de la sueur: kara ter "sueur noire", indiquant que le cheval ne pourrait parcourir qu'une courte distance au galop; žylma ter « sueur lisse ", légèrement scintillante et ačuu ter "sueur amère, salée ", abondante, qui montre que le cheval est prêt à courir sur n'importe quelle distance (Abduvaliev 1984, p. 7). Chez les Kazakhs, la sueur est dite successivement « écumeuse » (köbik ter), « âcre » (ašč ter ${ }^{27}$ ), puis « suave » (tättì ter). Quand la sueur devient moins salée, cela signifie que les muscles sont prêts à parcourir une longue distance.

Les Kazakhs de Mongolie distinguent quant à eux cinq sortes de sueur. La sueur "marécageuse" (batnaq ter) apparaît généralement dès les premières séances d'entraînement. Elle est ainsi nommée car elle se mélange à la saleté du poil. On l'ôte avec un couteau de chaleur. Cette première excrétion débarrasse la peau du cheval de ses impuretés. Ensuite la sueur est dite "écumeuse " (köbik ter). Après trois ou quatre séances d'entraînement, des flocons de sueur au goût salé apparaissent sous la bride et entre les cuisses. Cette deuxième excrétion débarrasse les parties du corps les plus grasses d'une humidité superflue. Ensuite la sueur est « aqueuse » (su ter). Cette sueur abondante et non salée comme de l'eau pure est émise par les parties musculeuses du cheval. Enfin la sueur " perlée » (monšaq ter) signale que la préparation du cheval est achevée. Elle apparaît en petites gouttelettes rondes et brillantes sur la croupe du cheval. On dit que «la sueur marécageuse vient de la peau, la sueur écumeuse vient de la graisse, la sueur aqueuse vient de la viande, la sueur perlée vient des os ». Les sudations successives sont donc censées avoir une origine de plus en plus profonde. Tous les éléments constituants du corps, du plus superficiel (la peau) au plus profond (les os), doivent perdre de leur humidité. Mais la sudation doit rester mesurée. Il existe dans ce système une cinquième sorte de sueur, dite "noire" (qara ter), excrétion abondante qui est le signe d'un entraînement excessif. Le cheval surentraîné se met à perdre du poids; à la vue de sa mine piteuse et de son poil trempé de sueur, le spécialiste le compare à " une souris sortie de l'eau » (sudan šyqqan tyšqandaj).

Afin d'exciter la sudation, les entraîneurs couvrent leurs chevaux pendant l'exercice. Selon Kaller, une dizaine de jours avant la course, on fait parcourir au cheval 4 à $5 \mathrm{~km}$, d'abord au pas, puis au trot, puis au galop, contre le vent, puis on lui met une couverture pour le faire suer davantage et on le travaille au trot. A la fin de la séance, on le fait marcher au pas sur une centaine de mètres, on l'attache dans un lieu couvert sans ôter la couverture et la sudation doit se poursuivre jusqu'à ce que la sueur perde son goût salée ${ }^{2}$. On lui ôte alors sa couverture et on continue à le mener en main jusqu'à ce qu'il sèche. Il passe la nuit attaché. Au matin, on lui donne un peu de foin, un peu d'avoine et de nouveau un peu de foin avant de l'abreuver à volonté. Enfin on le lave à l'eau froide, on le sèche puis on lui donne de nouveau du foin, de l'eau et $600 \mathrm{~g}$ d'avoine. 
Ce régime doit se poursuivre quotidiennement jusqu'à ce que toute graisse superflue soit éliminée (Kaller 1885, pp. 62-63).

\section{Séance d'entraînement au Kirghizstan. Un des chevaux est vêtu d'une couverture en feutre}
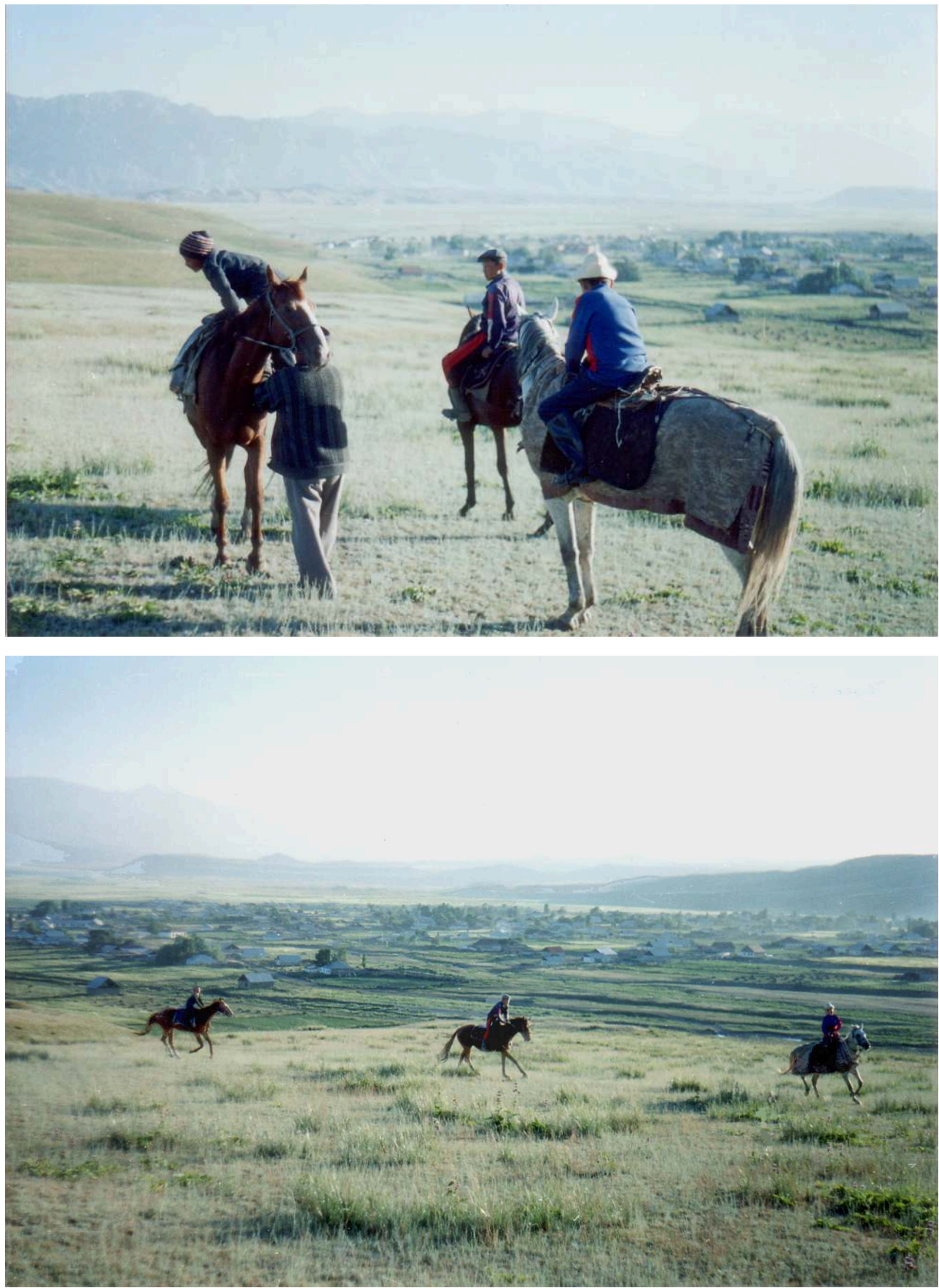

Photographies prises par C. Ferret en 1994 chez Obolbek Mamytov à Èmgekčil, arrondissement du Tjanšan région de Naryn, Kirghizstan 


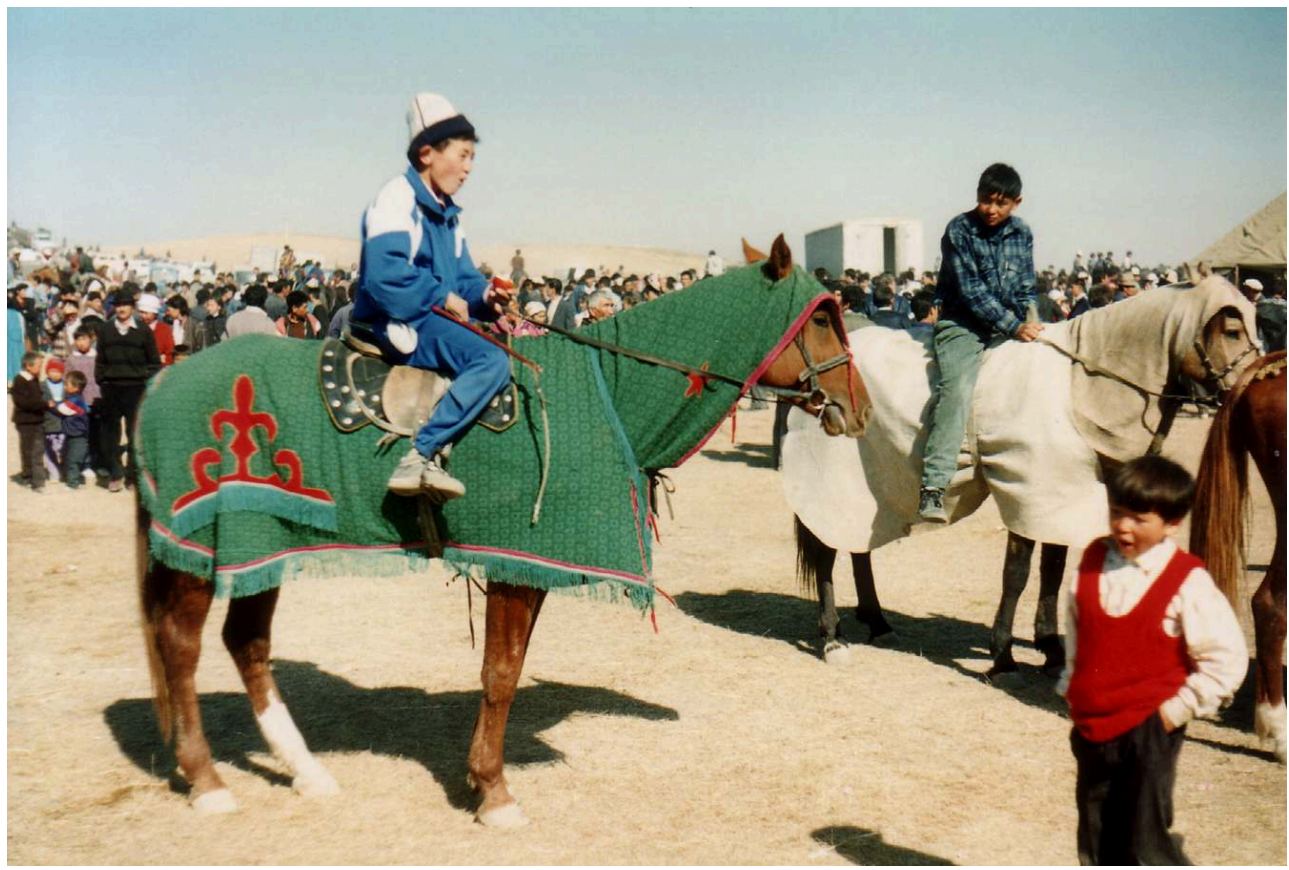

Photographie prise par C. Ferret le 16.10.1994 à Šien, arrondissement de Žambyl, région d'Almaty, Kazakhstan

51 Il ne faut pas confondre les deux fonctions des couvertures des chevaux de course kazakhs : les unes, en feutre, servent à provoquer la sécrétion de la sueur lors de l'entraînement ; les autres, plus fines, en tissu imprimé ou coloré, servent à dissimuler les formes du cheval pour le préserver du mauvais œil au moment d'arriver sur le lieu de la course et sont ôtées au départ. Elles se composent de deux parties : le qůlaqšyn (littéralement couvre-oreilles), cagoule couvrant la tête et l'encolure jusqu'au milieu du dos et le žabu, qui couvre le corps des épaules à la queue. Par ailleurs, beaucoup de chevaux de course portent en permanence un talisman (tůmar) autour de l'encolure, comme on en accroche au cou des enfants. 


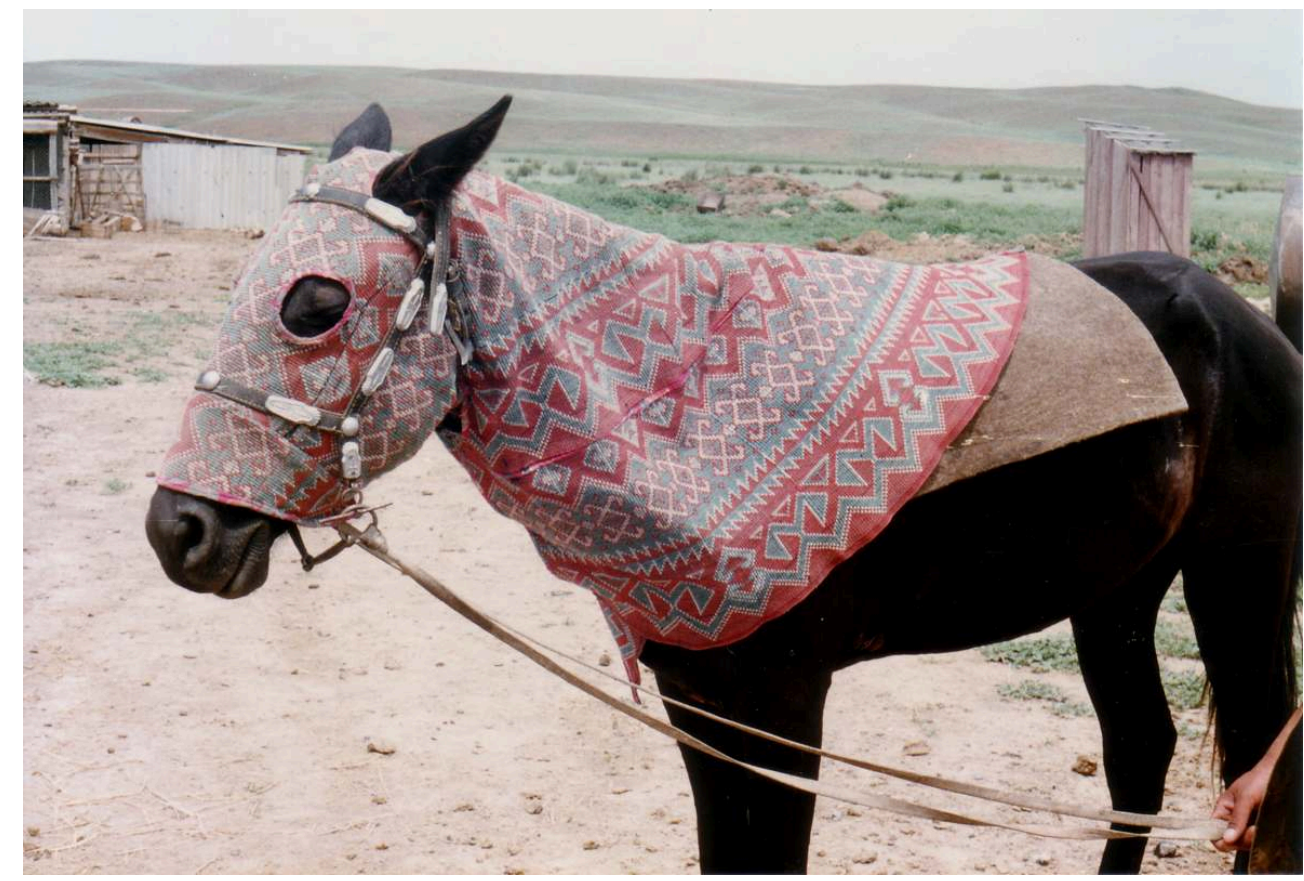

Qůlaqšyn et žabu sous une selle russe de cavalerie avec sanglage, poitrail, croupière, fouet et bridon kazakhs

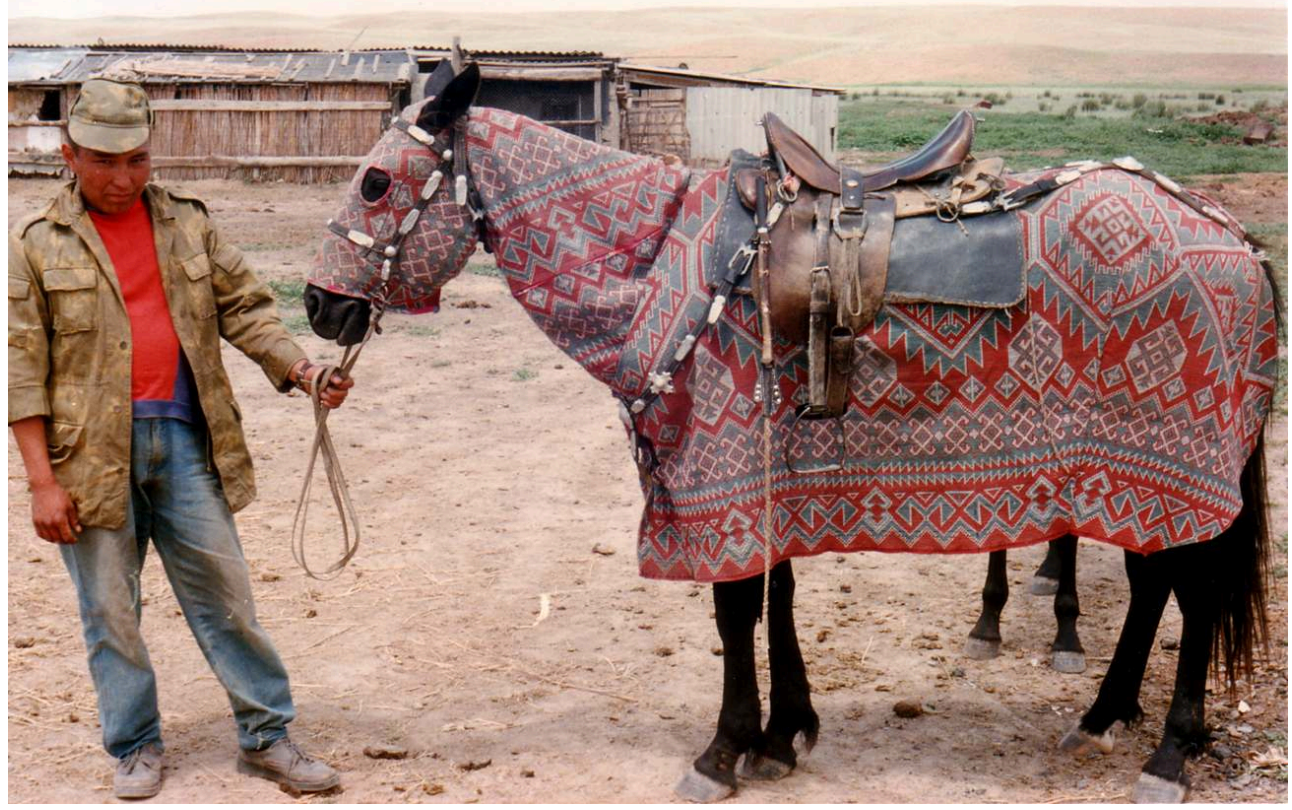

Photographies prises par C. Ferret en juin 1994 chez Melìs Alybekov à Tamgaly, arrondissement de Žambyl, région d'Almaty

Melìs Alybekov, jeune dentiste de la région d'Almaty (arrondissement de Žambyl) reconverti dans l'entraînement, attache son cheval à midi et le couvre à $5 \mathrm{~h}$. Il met d'abord un tissu fin, ter sorgyyš, qui absorbe la sueur, puis une grande couverture de feutre, kiìz, qui le couvre des oreilles à la queue, puis l'iškilik, tapis de selle en feutre troué à l'endroit du garrot, le žabu, puis un dernier tapis de selle nommé toqym avant de 
seller. Il lui fait faire 10 tours de $3,5 \mathrm{~km}$ au petit galop si c'est un cheval adulte, 5 ou 6 tours si c'est un poulain, jusqu'à ce que la sueur arrive aux sabots. Alors le cheval est rentré, il lui ôte ses couvertures, le conduit en main pendant une $1 / 2 \mathrm{~h}$ à $1 \mathrm{~h}$ pour le sécher, puis le garde attaché pendant $5 \mathrm{~h}$ si c'est un adulte, un peu moins pour les poulains. Le soir, il lui donne du foin, $1 \mathrm{~h}$ après de l'eau, puis trois jointées de blé pour les poulains, cinq pour les adultes. L'entraînement dure huit jours si le cheval est gras, cinq sinon.

Moldaraly Yrysov, entraîneur expérimenté dans l'arrondissement de Kegen (région d'Almaty), prépare ses chevaux aux courses de la manière suivante. Si le cheval est gras, il le fait d'abord travailler pendant deux mois comme simple cheval de selle, puis il l'entraîne pendant un mois. Dans le cas contraire, l'entraînement dure de deux semaines à un mois. L'exercice a lieu le soir, durant 2 à $3 \mathrm{~h}$. La première fois, il fait d'abord courir le cheval sans couverture, puis, une fois qu'il commence à suer, il l'enveloppe d'un kiiz pour accroître la sudation. Pendant la première demi-heure, il laisse le cheval aller à son rythme, car il a l'estomac encore plein. Puis, pendant une heure et demie, il le fait galoper à vive allure, sur un terrain accidenté. Il l'arrête lorsqu'il est uniformément couvert de sueur, lorsque « la sueur goutte du foie ». Alors il le fait marcher au pas sur $3 \mathrm{~km}$ et il le rentre en lui laissant sa couverture. Cette première séance s'appelle bìrinšì ter alu, "enlever la sueur une première fois ». Après l'entraînement, il attache le cheval à l'ombre. Il le laisse sans eau, sans nourriture et couvert toute la nuit. A $5 \mathrm{~h} \mathrm{du}$ matin, il lui donne $5 \mathrm{~kg}$ de foin, de l'eau et 2,5 $\mathrm{kg}$ d'avoine. Ensuite, il le lave soigneusement à l'eau et au savon pour enlever les traces de sueur. Puis il le laisse attaché à l'ombre toute la journée sans eau ni nourriture, dans un endroit propre et sec, couvert mais ventilé, où il peut se coucher. Ce jour-là, il ne le fait pas monter. Le lendemain, il le nourrit comme d'habitude le matin puis, à $5 \mathrm{~h}$ du soir, il lui donne un repas léger ( $2 \mathrm{~kg}$ de foin puis eau, puis $1 \mathrm{~kg}$ de foin) et il le couvre. A $7 \mathrm{~h} \mathrm{du}$ soir, il le fait monter, d'abord doucement pendant une demi-heure, " pour que le ventre se libère ", puis en le poussant au galop pendant une heure. Le cheval sait de lui-même quand il est fatigué et on l'arrête dès qu'il ralentit l'allure. Lors de cette deuxième séance, appelée ekìnš̀ ter alu "enlever la sueur une deuxième fois ", le cheval doit moins suer que la première fois. Si le cheval est gras, il lui faut sept séances de suée, effectuées tous les trois jours; s'il n'est pas gras, quatre ou cinq séances suffisent. A la suite de cet entraînement, le cheval doit avoir perdu son ventre. A ce moment-là, M. Yrysov ne lui met plus de couverture de feutre pour l'entraîner mais un simple tapis. Tous les trois jours, il le fait galoper sur une piste de $3 \mathrm{~km}$ en lui faisant faire 5 tours la première fois, 10 tours la deuxième, 15 la troisième. Après la première course, quand il l'entraîne pour la suivante, il ne lui fait plus subir que trois séances de suée car il retrouve plus rapidement la condition requise.

L'attache et la sudation permettent d'accélérer l'entraînement. Nůrtaj Sejdrahmanov, dans la région de Taldy-Qorgan, prépare les chevaux aux courses en deux semaines seulement. Après avoir attrapé son coursier dans le troupeau, il le garde attaché le premier jour. Le lendemain, il lui fait parcourir 4 à $5 \mathrm{~km}$ au trot sur un terrain sablonneux et l'attache sans boisson ni nourriture pour toute la nuit. Le matin, il le laisse paître pendant une heure. Le troisième jour, le cheval parcourt jusqu'à $15 \mathrm{~km}$ au trot sur un terrain sablonneux puis reste attaché. Le lendemain matin, il lui donne $2 \mathrm{~kg}$ de blé ou d'avoine humectée. Le quatrième jour, il le fait galoper 15 à $20 \mathrm{~km}$ pour le faire suer, séance qui s'appelle qara žarys " course noire " ${ }^{29}$. Ensuite il goûte sa sueur 
pour voir si elle est amère ou sucrée. Si la sueur est amère, il habille le cheval d'une lourde couverture de feutre et il lui fait parcourir tous les jours 8 à $10 \mathrm{~km}$, tantôt au trot tantôt au galop. La nuit, il lui enlève la couverture et il l'attache. Le matin, il lui donne $2 \mathrm{~kg}$ de céréales. Après cet entraînement, la sueur devient légèrement sucrée, signe que le cheval est prêt.

Pour enlever les traces de sueur dues à ces séances de sudation, les entraîneurs passent après l'exercice un couteau de chaleur appelé terì qyrg̀yš, qui peut aussi être un simple couteau de cuisine utilisé du côté non tranchant, afin que le sel sorti avec la sueur ne pénètre pas de nouveau dans le corps du cheval. Les chevaux sont rarement pansés, mais souvent lavés à grande eau par beau temps, avec ou sans savon, ou baignés une fois par semaine dans une rivière. Quand le climat est plus froid, on se contente éventuellement de doucher les membres et de nettoyer le corps à l'éponge humide.

Les entraîneurs observent également le moment où le cheval sue (au cours de l'exercice ou quelques heures après celui-ci), car mieux le cheval est entraîné, plus la sueur sort tardivement, et ils veillent à ce que la sudation soit bien uniforme : le corps doit être entièrement couvert de sueur, sans zones sèches qui seraient douloureuses le lendemain. Selon Müsì̀̀m Äbìkov, entraîneur dans l'arrondissement de Žarma (région de Semej), la sueur doit rouler en gouttes rondes, sans laisser de traces, alors que sur certains chevaux, les marques de sueur forment des lignes divergentes car leur propriétaire, à tort, les nourrit uniquement de foin, sans herbe ni céréales.

Les entraîneurs kazakhs considèrent que l'excrétion de sueur soulage le cheval, en le débarrassant à la fois de son eau et de son sel. Après quelques séances de sudation, le cheval respire moins lourdement à l'entraînement. D'une part, on extrait le sel du corps du cheval en le faisant suer et d'autre part, on se garde de distribuer du sel aux chevaux à l'entraînement parce que c'est un élément qui retient l'eau et ramollit les muscles. En kazakh, enlever la sueur (ter alu) est synonyme d'entraîner, ce qui montre bien l'importance de la sudation dans la préparation aux courses.

\section{Les signes de la complétude}

Outre le goût de la sueur, d'autres indices révèlent la condition du cheval à l'entraînement. Selon les informateurs du Semireč'e, un cheval en forme doit déféquer rarement, son crottin doit être sec et en petite quantité ; c'est le signe d'un bon état physiologique. Dans l'Est kazakh, Näcìbolda Nůrsipov attend que le crottin devienne sec, indice que «les intestins ne sont pas détendus ». Quand il serre un crottin dans son poing, rien ne doit dépasser entre les doigts, sinon cela signifie que le cheval n'est pas prêt. Mais dans la région de Pavlodar, Můqataj Qůlžanov exprime une opinion contraire : « ceux qui ne savent pas entraîner les chevaux les mènent à un état où leur crottin devient sec (qu qůmalaq istedi). Quand on serre du crottin dans la main, il doit rester des petites taches sur la paume ». Il juge qu'un cheval est prêt quand ses muscles et sa peau se développent et s'affermissent uniformément (et pen terì tolyp, bìrdej qatu kerek).

59 On observe également la couleur de l'urine, qui doit progressivement s'éclaircir, jusqu'à devenir transparente. A l'inverse, l'urine d'un cheval surentraîné secrétant de la sueur "noire " prend la teinte du thé ; on dit alors qu'il "pisse le sang " et ses crottins s'amollissent. 
60 Selon Dobrosmyslov, pour vérifier la condition du cheval, on le monte pendant 15 à $20 \mathrm{~km}$, puis on descend et on lui donne aussitôt à boire. S'il se contente de deux gorgées et cesse aussitôt de s'abreuver, c'est signe qu'il est prêt (1895, p. 127). De même, un entraîneur de la région de Qyzylorda emmène son cheval paître tôt le matin de la course, à un endroit où pousse de la fétuque des moutons (Festuca Ovina). Au moment où le cheval ne broute plus toute l'herbe avec sa tige, mais choisit sa nourriture, c'est-àdire se contente de manger la pointe, il l'emmène à l'abreuvoir. Si, après avoir bu deux gorgées, il se détourne de l'eau et regarde de côté, c'est signe qu'il est en bonne forme et qu'il est prêt à participer à la compétition, mais s'il boit sans discontinuer, cela signifie que le processus d'entraînement n'est pas encore mené à son terme et qu'il faut remettre la compétition à un autre jour.

61 Les critères principaux permettant de juger que le cheval est prêt à participer à une épreuve sont donc liés au sec et à l'humide : le crottin doit être sec, l'urine claire et l'animal doit boire peu. Le cheval à l'entraînement doit être comme asséché, mais asséché de telle manière qu'il ne ressente pas le besoin de boire, son organisme s'étant adapté à cette sobriété.

\section{Derniers préparatifs}

62 Selon Kaller, on attache court le cheval pendant les derniers jours qui précèdent la course pour qu'il ne puisse pas se coucher $(1885$, p. 63). De nombreux entraîneurs confirment ces affirmations et déclarent passer eux-mêmes la dernière nuit à l'écurie pour empêcher leur cheval de dormir. Seul un entraîneur de la région d'UstKamenogorsk fait une litière propre à son cheval la veille de la course et lui donne 2 à 3 kg d'avoine le soir " pour qu'il se couche et dorme bien ».

63 En ce qui concerne l'exercice et la nourriture à la veille de la course, les avis sont partagés. Selon Dobrosmyslov, on ne monte pas le cheval et on ne le nourrit pas la veille et le jour des courses; on l'abreuve seulement en lui donnant éventuellement sa ration habituelle de lait (1895, p. 127). De même, un entraîneur de la région de TaldyQorgan ne nourrit pas son cheval deux jours avant la compétition. Il lui donne seulement un peu de foin la veille au soir et deux jointées d'avoine pure lavée le matin de la course. Mais on considère souvent qu'un cheval qui a le ventre vide ne peut pas courir longtemps. C'est pourquoi beaucoup nourrissent néanmoins leur coursier en restant fidèle au type d'aliments distribués pendant l'entraînement. Moldaraly Yrysov donne le soir $1 \mathrm{~kg}$ de foin avant d'abreuver et $2,5 \mathrm{~kg}$ après. Il garde son cheval attaché pendant deux heures, puis lui donne $15 \mathrm{~kg}$ de foin. Le matin de la course, à $4 \mathrm{~h}$, il lui donne $0,5 \mathrm{~kg}$ de foin avant de l'abreuver, $2 \mathrm{~kg}$ après et plus rien jusqu'à la course. La veille de la course, il l'entraîne avec modération pendant la journée et non le soir comme d'habitude. D'autres entraîneurs laissent brouter leur cheval pendant une demi-heure.

De nos jours, les chevaux sont amenés sur le lieu de la course en camion ouvert, mais à l'abri des regards malveillants sous leur couverture. Quand la course est annoncée, on attache le toupet avec un morceau de tissu de façon à ce qu'il soit dressé en l'air comme une houppe (kekil); on plie la queue et on la bande avec un morceau de tissu assorti tout en laissant une fine mèche longue. Il ne reste plus à l'entraîneur qu'à donner les dernières recommandations à l'enfant jockey qui se dirige vers la ligne de départ... 


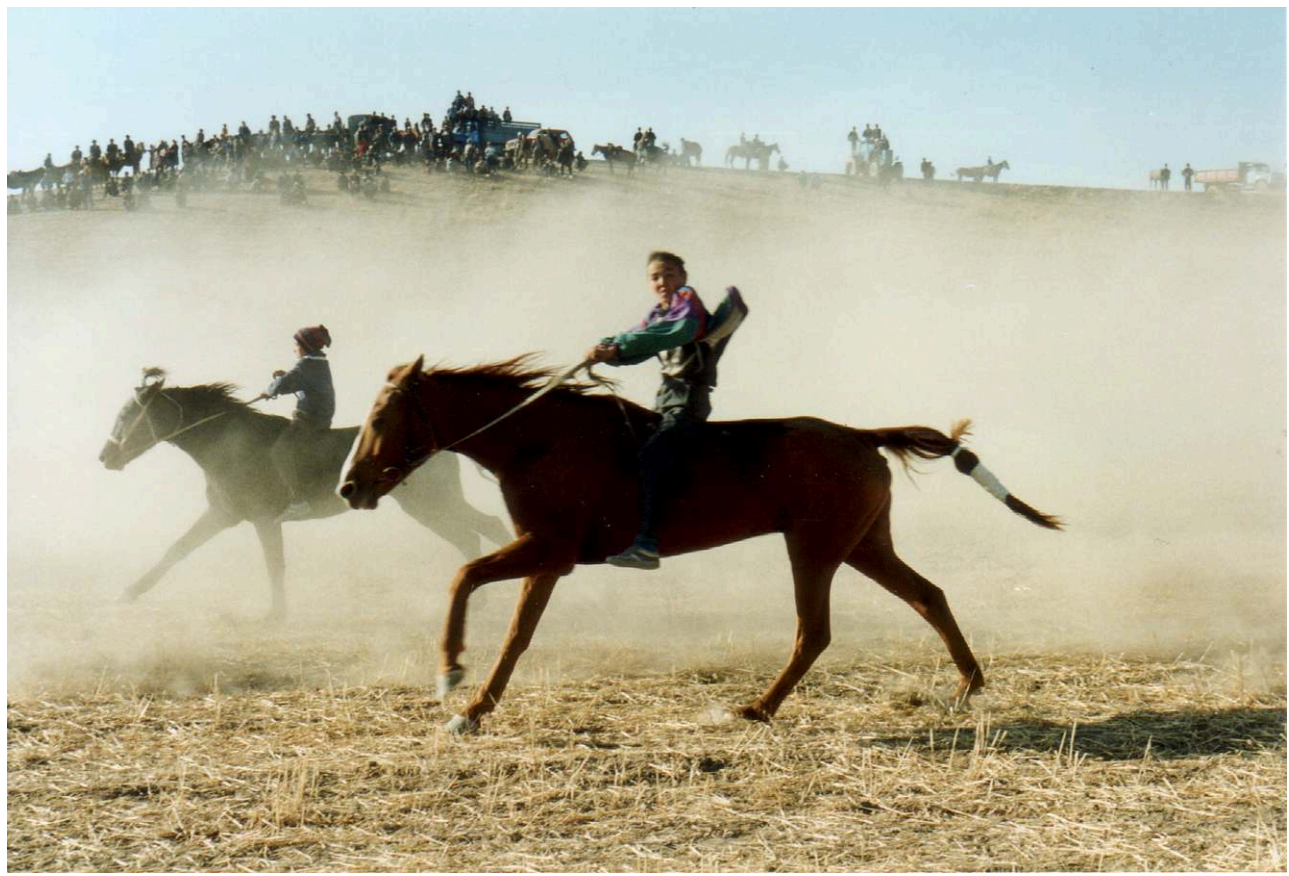

Photographie prise par C. Ferret le 16.10.1994 à Šien, arrondissement de Žambyl, région d'Almaty

\section{Conclusion}

Cet article, issu d'études de terrain, a pour seule ambition d'apporter quelques renseignements sur une technique totalement méconnue en Occident. L'analyse de ces données permettra de mieux comprendre quelles représentations de la physiologie animale elles mettent en $\mathrm{jeu}^{30}$ et de les comparer avec d'autres méthodes d'entraînement employées pour d'autres types de compétitions équestres (le modèle esthétique des chevaux kazakhs de kökpar - l'équivalent du bozkachi afghan - est tout autre et leurs méthodes d'entraînement diffèrent également) ou issues d'aires culturelles proches (Mongolie ${ }^{31}$, Turkménistan) ou lointaines (Occident). Par exemple, il est curieux de remarquer qu'au XIX $x^{e}$ siècle, on excitait également la sudation des pursang anglais en les faisant courir entièrement couverts, ou même en leur faisant prendre des bains de vapeur. Ces parallèles méritent d'être étudiés plus en détail.

D'ores et déjà, l'exposé du détail des opérations liées à l'entraînement du cheval de course chez les Kazakhs nous permet de conclure qu'au-delà des divergences pratiques et théoriques, l'ensemble de cet entraînement peut être interprété comme une cure d'amaigrissement ou comme un processus d'assèchement, assécher signifiant ici ôter l'humidité et la graisse. Pour comprendre ces méthodes d'entraînement, il est nécessaire d'examiner la manière dont elles s'intègrent dans les représentations symboliques du sec et de l'humide, du chaud et du froid. La sueur, humidité chaude dont la sécrétion permet d'extirper la graisse et l'eau du corps du cheval, ne revêt pas, chez les Kazakhs, la même connotation laborieuse qu'en Occident.

Une première interprétation tendrait à montrer que cette cure d'amaigrissement a pour objet d'alléger le cheval. C'est en effet dans le même souci de légèreté qu'on fait monter les chevaux par des enfants, sans selle et même souvent pieds nus. Et ce n'est 
pas un hasard si certains entraîneurs donnent à leurs chevaux des noms d'oiseaux, ces chevaux dont on dit aussi qu'ils sont les ailes de leurs héros. Mais cette première interprétation est insuffisante parce qu'elle reste muette sur l'origine de la force dont le cheval doit user pour parcourir au galop une si longue distance : la légèreté ne suffit pas. En outre, on ne peut que s'étonner de l'acharnement de cette lutte contre la graisse, menée dans une culture où cet élément est par ailleurs tant valorisé, notamment dans l'alimentation des hommes. En fait, il ne s'agit pas tant de faire disparaître la graisse que de la déplacer vers l'intérieur, en la "transformant » en moelle ${ }^{32}$, puisque, selon les Kazakhs, le cheval tire sa force de sa moelle osseuse et non de ses muscles. L'exposé du détail des techniques d'entraînement lève donc le voile sur une physiologie originale dont le champ d'application dépasse largement le cadre de l'équitation ${ }^{33}$.

\section{BIBLIOGRAPHIE}

Abduvaliev, Ibraim

1984 Konnosportivnaja leksika v kirgizskom jazyke [Le lexique kirghiz des sports hippiques], Avtoreferat dissertacii na soiskanie učenoj stepeni kandidata filologičeskih nauk (Frunze).

Babadžanov, Hodži Salih

1871 Lošadi i ih ispytanija vo vnutrennej kirgizskoj orde [Les chevaux et les courses dans la horde intérieure kirghize], Žurnal konnozavodstva [Le journal des haras], XXX (5-6-7), pp. 21-37 ( $\left.{ }^{\circ} 5\right)$;

$35-48\left(n^{\circ} 6\right) ; 63-87\left(n^{\circ} 7\right)$.

Barmincev, Ju. N.

1958 Evoljucija konskih porod v Kazahstane. Opyt zootehničeskogo issledovanija problemy porodoobrazovanija [L'évolution des races équines au Kazakhstan. Essai d'étude zootechnique du problème de la formation des races] (Alma-Ata, Kazgoizdat).

Benkevič, V.

1905 Nabljudenija, materjaly i zametki, sobrannye v Kirgizskoj stepi [Observations, données et remarques rassemblées dans la steppe kirghize], Vestnik obščestvennoj veterinarii [Le messager de la médecine vétérinaire publique], XVII (23).

Dobrosmyslov, A. I.

1895 Skotovodstvo $v$ Turgajskoj oblasti [L'élevage dans la région de Turgaj] (Orenbourg, Izdanie Turgajskogo oblastnogo statističeskogo komiteta).

Džantjurin, C.

1883 Očerki kirgizskogo konevodstva [Précis d'élevage équin kirghiz] (Orenbourg).

Ferret, Carole

1997 Les courses de chevaux en Asie centrale, La Lettre d'Asie centrale, 6, pp. 8-10.

2004 De l'attache des chevaux à la fécondation des femmes en passant par la cuisine. Quelques pistes pour l'exploration des notions altaïques de chaud et de froid, Études rurales, 171-172, pp. 243-270.

2006 Techniques iakoutes aux confins de la civilisation altaïque du cheval. Contribution à une 
anthropologie de l'action. Thèse de doctorat en anthropologie sociale et ethnologie (EHESS, Paris), 3 vol.

2008 Course à la mort ou quête de respectabilité : le bäjge en Asie centrale, Ethnozootechnie, $n^{\circ} 82$ [Histoire des courses, journée d'études du 6 décembre 2007], pp. 129-148.

2009a Une civilisation du cheval. Les usages de l'équidé de la steppe à la taïga (Paris, Belin).

2009b Des chevaux pour l'empire, Les Cahiers d'Asie centrale, n 17-18 [Le Turkestan russe : une colonie comme les autres? dirigé par S. Gorshenina \& S. Abashin], pp. 211-253.

Kaller, A.

1885 Kirgizskij sposob prigotovlenija lošadej k skačkam vo Vnutrennej kirgizskoj Bukeevskoj orde [La méthode kirghize de préparation des chevaux aux courses dans la horde intérieure de Bökej], Žurnal konevodstva [Le journal de l'élevage équin], n 3, pp. 57-64.

Marcenac, L.-N., H. Aublet et P. d'Hauteville

1974 [1964] Encyclopédie du cheval, (Paris, Maloine).

Meserve, Ruth I.

1993 The Traditional Mongolian Method of Conditioning Horses and Preventive Veterinary Medicine, in International Symposium of Mongolian Culture (Taipei, ROC), pp. 484-501.

Simakov, G. N.

1984 Obščestvennye funkcii kirgizskih narodnyh rasvlečenij v konce XIX - načale XX v. (istorikoètnografičeskie očerki) [Les fonctions sociales des divertissements populaires kirghiz de la fin du $\mathrm{XIX}^{\mathrm{e}}$ et du début du XX $\mathrm{X}^{\mathrm{e}}$ siècle (Essai historico-ethnographique)] (Leningrad, Nauka).

1980 Hozjajstvo kazaxov na rubeže XIX-XX vv. [L'économie des Kazakhs au tournant des XIX ${ }^{\mathrm{e}}$ et XX siècles] (Alma-Ata, Nauka).

\section{ANNEXES}

\section{Informateurs}

Région de Taldyqorgan (AT) :

- Nưrtaj Sejdrahmanov (Žalayr), né en 1922

Région de Pavlodar (AT) :

- Můqataj Qůlžanov, âgé de 70 ans

Région d'Aqtöbe (AT) :

- Sarin Sůltangali, arrondissement de Qarabůtaq

Région du Sud kazakh (CF) :

- Anarbek Qožabekov (Ůly žüz > Dulat > Žanys > Šegìr), né en 1938.

- Tìleubek Älìmtaev (Ůly žüz > Qaply > Šoqpar), né en 1962.

- Ämìr Tůraev (Ůly žüz > Suan > Bag̉ys), né en 1915.

Région de Žambyl (CF) :

- Žanybek Alybekov (Uீly žüz > Aqqoily), né en 1953, entraîneur de chevaux au haras de Lugovoe. Frère cadet de Zaurbek Alybekov, il travaille au sovkhoze depuis 1976.

- Zaurbek Alybekov (Ůly žüz > Aqqoily), directeur de la section d'élevage équin du sovkhoze (haras) de Lugovoe.

- Eduard Kazbekovič Gudiel, russe, entraîneur au haras de Lugovoe. Il s'est occupé pendant 18 ans de l'entraînement aux courses classiques des chevaux akhal-tékés du haras. 
Région d'Almaty (CF) :

- Melìs Alybekov, né en 1967, dentiste puis éleveur et entraîneur de chevaux, arrondissement de Žambyl.

- Ġabit Qůralbaev (Šaprašty > Ekej), né en 1959, entraîneur, arrondissement de Žambyl. Cela fait 9 ans qu'il travaille avec les chevaux de course, d'abord comme jockey puis comme entraîneur.

- Bolat Razaqov, ouïgour, né en 1970, entraîneur de chevaux, arrondissement de Kašlen.

Rajymbekskij rajon (arrondissement de Kegen, dans la région d'Almaty) (CF):

- Moldarälì Yrysov, né en 1931, Kegen. Cela fait 50 ans qu'il entraîne des chevaux à des courses de longues distances, soit depuis l'âge de 17 ans.

- Nůrbol Malybekov, né en 1970, vacher, entraîneur de chevaux à Aqtas.

Région de Semipalatinsk (kaz. Semeu) (CF \& AT) :

- Müsìlìm Äbìkov, né en 1936, berger et zootechnicien, arrondissement de Žarmin. Il a vécu en Chine jusqu'en 1962.

- Säbidolla Můqaůly (Orta žüz > Kerej > Qůrman), né en 1949 en Mongolie, où il habite toujours, rencontré près d'Üš Bì̀k.

- Qajdolla Můqaůly (Orta žüz > Kerej > Qůrman), né en 1961 en Mongolie, où il habite toujours, rencontré à Üš Biìk. C'est le frère cadet de Säbidolla Můqaůly.

Région de l'Est kazakh (CF) :

- Näsìbolda Nůrpeiìsov (Kerej), né en 1928, retraité, ancien rus. tabunščik « gardien de troupeaux de chevaux » et berger. Il a travaillé de 1947 à 1952 comme tabunščik, puis ensuite comme berger jusqu'en 1965, et a reçu diverses décorations à ce titre. Il a dix enfants.

\section{NOTES}

1. Les sources russes écrivent bajga. Les ethnonymes sont écrits en transcription francisée, les noms propres de personnes et de lieux sont translittérés à partir de leur orthographe kazakhe.

2. Le nauryz kazakh équivaut au nawrūz persan, premier jour de l'année solaire.

3. Sur différents types de courses hippiques pratiqués par les peuples turco-mongols d'Asie centrale et de Sibérie, $c f$. Ferret 2009a.

4. Littéralement, ce mot signifie « soulever de terre".

5. Les Kazakhs sont organisés en trois žüz « horde ", à savoir ůly žüz « grande horde » ou « horde aînée ", dans le Sud-Est ; orta žüz « horde moyenne ", dans le centre ; kiš̀ žüz " petite horde » ou « horde cadette », dans le Nord-Ouest et l'Ouest.

6. Nous traduisons les divisions administratives territoriales rus. oblast' (kaz. oblys)par " région " et, au niveau inférieur, rus. rajon (kaz. audan) par « arrondissement ».

7. La horde de Bökej (rus. Bukej), appelée horde intérieure à partir de 1845, a été formée après qu'un groupe de Kazakhs de la petite horde a migré sur un territoire situé entre l'Oural et la Volga.

8. Qarǵy signifie « sauter, bondir ».

9. Qašaǵan signifie « farouche, sauvage, fuyant ».

10. Au sujet des instruments de capture des chevaux, voir Hozjajstvo kazahov 1980, p. 110 et Ferret 2006, pp. 428-438.

11. $A u$ XIX ${ }^{e}$ siècle, les Russes appelaient les Kazakhs actuels Kirghiz-Kaïssaks, ou bien simplement Kirghiz, et les Kirghiz actuels Kara-Kirghiz. 
12. Littéralement « zibeline noire».

13. Suspendre le crâne de certains chevaux particulièrement prisés est une coutume encore pratiquée chez les peuples turcophones ( $c f$. Ferret 2009a).

14. Littéralement " gazon noir ", manière de désigner un noir profond. Les chevaux kazakhs sont souvent nommés d'après leur robe.

15. La "bosse du saut» est une saillie observée chez certains chevaux entre les hanches, à la jonction des vertèbres lombaires et sacrées, qui est censée favoriser l'aptitude au saut d'obstacles.

16. La croupe est dite avalée, ou « en pupitre », lorsqu'elle est très oblique, sa ligne formant un angle de plus de $45^{\circ}$ avec l'horizontale.

17. De façon générale, on trouve peu de comparaisons animales dans la description des beautés et défectuosités, contrairement à ce qu'on remarque en Mongolie ou même en France. L'exception que constitue cet exemple vient peut-être de ce que les informateurs étaient des Kazakhs originaires de Mongolie.

18. Un cheval fait « en descendant » a le garrot plus bas que la croupe.

19. Pour une interprétation des considérations thermiques de l'attache $c f$. Ferret 2004.

20. Kaz. qazy est aussi le nom d'un saucisson fait avec de la graisse abdominale et costale du cheval.

21. Les Kazakhs mesurent les céréales par jointées, soit le contenu de deux mains jointes.

22. Semireč'e : région dite des sept rivières, au Sud-Ouest du Kazakhstan.

23. As : fête célébrant le souvenir d'un défunt et ayant lieu, en principe, un an après la mort.

24. A titre indicatif, en Europe, on recommande de donner quotidiennement à un cheval de $500 \mathrm{~kg}$ soumis à un travail léger $7,5 \mathrm{~kg}$ de foin et $2,5 \mathrm{~kg}$ de céréales et, s'il est soumis à un travail intense, $5 \mathrm{~kg}$ de foin et 7,5 kg de céréales (cf. Marcenac, Aublet et Hauteville 1974 [1964], p. 364), alors que, chez cet entraîneur kazakh, un cheval au repos reçoit $16 \mathrm{~kg}$ de céréales et 24 à $40 \mathrm{~kg}$ de foin.

25. En France, on respecte le même ordre, bien que la justification soit différente : il faut abreuver après le foin pour débarrasser des poussières et avant le grain pour éviter les gonflements d'estomac. Néanmoins, l'avoine est également dite « échauffante ».

26. Par rapport aux courses de chevaux kazakhes, les compétitions kirghizes présentent quelques petites différences : les prix sont moins importants, les distances parfois plus réduites, les poulains ne courent pas avant l'âge de deux ans et les concurrents montent généralement avec une selle. Mais l'entraînement est fondé sur les mêmes principes.

27. En kazakh, aščy désigne un goût qui peut être à la fois salé, amer ou acide.

28. Selon tous les entraîneurs actuels, la sueur ne perd sa salinité qu'après plusieurs séances.

29. Le qara žarys est traditionnellement une course de moindre distance, sans prix, organisée la veille d'une alaman bäjge.

30. C'est ce que j'ai fait dans un autre article, paru précédemment du fait des aléas de l'édition (Ferret 2004).

31. Sur les méthodes mongoles d'entraînement des chevaux, fort proches des kazakhes, voir Meserve 1993 ainsi que l'article de G. Lacaze dans le présent recueil.

32. La moelle se dit en kazakh süjek majy, littéralement graisse des os.

33. Cette question est plus amplement développée dans Ferret 2004. 


\section{RÉSUMÉS}

À partir de matériaux de terrain recueillis dans les années 1990, les auteurs exposent les critères de sélection des chevaux de course kazakhs pour les compétitions de longue distance (kaz. bäjge) et décrivent les détails de leurs méthodes d'entraînement, qui visent à l'allègement des coursiers et révèlent l'existence d'une physiologie centrasiatique originale, marquée par l'opposition du chaud et du froid, du sec et de l'humide.

Based on field research lead by both authors in the 1990's, this paper examines the selection criteria of Kazakh racehorses over long distances and describes in detail Kazakh training methods, which aim to make racehorses lighter, and reveal an original Central Asian physiology, characterized by the opposition of hot and cold, wet and dry.

\section{INDEX}

nomsmotscles Kazakh

Keywords : body, feast, horse, horse races, hot and cold, physiology, sweat, training, wet and dry Mots-clés : chaud et froid, cheval, corps, courses, entraînement, fêtes, physiologie, sec et humide, sueur

Index géographique : Kazakhstan

\section{AUTEURS}

\section{CAROLE FERRET}

Carole Ferret, docteur en ethnologie et anthropologie sociale, chercheur à l'Institut français d'études sur l'Asie centrale (Tachkent) et chercheur affilié au Laboratoire d'anthropologie sociale (Paris), auteur, entre autres, de Une civilisation du cheval. Les usages de l'équidé de la steppe à la taïga (Paris, Belin, 2009).

\section{AHMET TOQTABAEV}

Ahmet Toqtabaev (Toqtabaj), ethnographe, chercheur à l'Institut d'histoire et d'ethnologie Č. Č. Valihanov (Almaty), titulaire d'une thèse de $3^{\mathrm{e}}$ cycle sur l'élevage kazakh du cheval aux XIX $\mathrm{xx}^{\mathrm{e}}$ siècles (1992) et auteur, entre autres, de Kul't konja u kazahov [Le culte du cheval chez les Kazakhs] (Almaty, Kazizdat, 2004). 